'Departamento de Medicina Familiar, Facultad de Medicina, Pontificia Universidad Católica de Chile. Santiago, Chile. ${ }^{a}$ Asesor estadístico Master in Business Administration. Massachusetts Institute of Technology, Boston, USA.

Trabajo finaciando por: Fondo Concursable Becarios, Dirección de Investigación, Escuela de Medicina, Pontificia Universidad Católica de Chile. Los autores declaran no tener conflictos de interés.

Recibido el 9 de septiembre de 2018, aceptado el 28 mayo de 2019.

Correspondencia a: María Victoria Rodríguez mvrodriguez1@uc.cl

\section{Percepciones y actitudes de médicos de atención primaria respecto de las Guías de Práctica Clínicas en el Régimen de Garantías en Salud}

\author{
MARÍA VICTORIA RODRÍGUEZ ${ }^{1}$, FLORENCIA PRIETO', \\ TOMÁS PANTOJA ${ }^{1}$, FRANCISCO LETELIER ${ }^{\mathrm{a}}$
}

\section{Perceptions and attitudes of Chilean primary care physicians toward clinical practice guidelines}

Background: Clinical practice guidelines (CPGs) promote better quality and equity in health care and potentially they could improve patients' outcomes. However, their implementation is hindered by a number of factors including some related to health care professionals. Aim: To assess the perceptions and attitudes of primary care physicians regarding CPGs developed by the Chilean Ministry of Health in the context of the Health Sector Reform. Material and Methods: An adaptation of the survey "Knowledge, perceptions and attitudes towards Clinical Practice Guidelines" was sent to 1,264 primary care physicians in Chile and answered completely by 354. The analysis assessed the attitudes towards CPG, their use in primary care and their relationship with socio demographic features of respondents. Results: Eighty two percent of respondents reviewed the flowcharts of the guidelines, $85 \%$ consulted their online version. The classification of evidence levels and the strength of recommendations generated a high level of confidence with the guidelines in 70 and $64 \%$ of respondents. Eighty five percent considered that $C P G$ could help to standardize clinical practice. The most relevant barrier hindering CPG use was the lack of a brief, simple and easy to access format in $63 \%$ of respondents. The three dimensions of the theory of planned behavior (attitude toward behavior, subjective norms, and perceived behavioral control) were associated with a greater frequency of guideline use. A higher age and not being Chilean were associated with a lower frequency of use. Conclusions: The identified factors associated with CPG use should be considered in future guideline design.

(Rev Med Chile 2019; 147: 602-611)

Key words: Chile; Practice Guideline; Primary Health Care.
$\mathrm{L}$ as guías de práctica clínica (GPC) son "enunciados elaborados sistemáticamente para asistir las decisiones de los profesionales sanitarios y sus pacientes acerca del cuidado apropiado en circunstancias clínicas específicas" Asimismo, su objetivo es promover una mayor calidad en la asistencia sanitaria ${ }^{2-4}$. Su principal finalidad es trasladar el conocimiento científico a recomendaciones que ayudarán al clínico a disminuir su incertidumbre en la toma de decisiones ${ }^{5}$.

Una adecuada implementación de las GPC podría mejorar los resultados sanitarios de los 
pacientes ${ }^{6,7}$. Sin embargo, su efectividad para cambiar la práctica clínica ha sido variable ${ }^{6}$, existiendo brechas significativas entre las recomendaciones presentadas en ellas y su uso en la práctica clínica ${ }^{8}$. Esta brecha podría deberse a diversos factores, que pueden ser agrupados en tres áreas: (a) relacionados a características propias de las GPC; (b) relacionadas con los usuarios de las GPC (características psicológicas y sociodemográficas; conocimiento, actitud y conformidad con las recomendaciones) y (c) relacionados con las organizaciones de salud dónde son aplicadas?.

Respecto de aquellos factores relacionados con los usuarios de las GPC, existe una serie de estudios que exploran sus percepciones respecto de ellas ${ }^{10-14}$. Para la mayoría de los clínicos, las GPC son una valiosa fuente de asesoramiento y formación, y mejoran la calidad de atención sanitaria. Sin embargo, algunos consideran que su objetivo es reducir costos sanitarios, y que son rígidas, destacando la dificultad para aplicarlas a pacientes individuales y su potencial de conducir a demandas y toma de decisiones equivocadas.

En Chile, desde el 2005 se viene implementando el Régimen de Garantías Explicitas de Salud (GES) y uno de los elementos centrales de esta política ha sido la elaboración, diseminación e implementación de GPC ${ }^{15}$.

Considerando, la relevancia de los factores relacionados con los clínicos en la implementación de las GPC, y dado que un considerable número de ellas están dirigidas a la Atención Primaria (AP), el presente estudio tiene como objetivo determinar las percepciones y actitudes de médicos de atención primaria acerca de GPC del Régimen de GES, a través de la teoría del comportamiento planeado, con el fin de contribuir al diseño de estrategias de implementación más efectivas para ellas en la AP de nuestro sistema de salud. Como objetivo específicos se describen las características de los usuarios encuestados evaluando los factores que pudieran estar asociados a una mayor frecuencia de uso de las guías.

\section{Material y Método}

Estudio descriptivo de corte transversal, basado en la aplicación de un cuestionario a médicos que trabajaban en AP.

\section{Elaboración del cuestionario}

Se realizó una adaptación del cuestionario "Conocimientos, percepciones y actitudes frente a las Guías de Práctica Clínica"14,16 elaborado con técnicas cualitativas y cuantitativas con el objetivo de explorar cuáles eran las percepciones y las actitudes de profesionales españoles hacia las GPC. La adaptación consistió en modificaciones mínimas principalmente relacionadas a aspectos del contexto. Se realizaron dos pruebas pilotos, con 16 médicos de dos Centros de Salud de la Región Metropolitana de Santiago evaluando la compresión, claridad y aceptación del instrumento. El cuestionario final incluyó 24 preguntas, agrupadas en cinco dominios: datos demográficos, actividad laboral, participación en elaboración de guías, percepciones y actitudes de GPC GES y frecuencia de uso (Anexo 1).

Las preguntas del dominio percepciones y actitudes, estuvieron organizadas de acuerdo a la teoría del comportamiento planeado (TCP $)^{17}$. La TCP propone tres aspectos ("dimensiones") que estarían relacionados con la intención de realizar una conducta y con la conducta propiamente tal (en este caso la conducta propuesta por la recomendación de la GPC). Estas dimensiones son: actitud, que refleja la evaluación que la persona hace sobre la conducta; norma subjetiva, que resulta de la evaluación que se hace sobre si los otros significativos desean que la conducta se realice y de la motivación para complacerlos; y control percibido, que consiste en la percepción del individuo sobre su capacidad de realizar la conducta (Figura 1) ${ }^{18-20}$. Estas 3 dimensiones fueron medidas utilizando preguntas con escala de respuestas tipo Likert de 7 puntos (1 "totalmente en desacuerdo" y 7 "totalmente de acuerdo").

La conducta (variable dependiente), fue medida a través de preguntas del dominio "frecuencia de uso de las guías", categorizada en 5 grupos (la mayor parte de los días, 2-3 veces por semana, 1 vez por semana, algunas veces, y ninguna vez). Para el análisis esta variable fue dicotomizada en: (i) aquellos que usan las guías 1 o más veces por semana; y (ii) aquellos que las usan menos de una vez por semana.

\section{Muestra y recolección de la información}

Tomando como punto de partida una base de datos del Ministerio de Salud (MINSAL) que identificaba 3.831 médicos trabajando en AP, se 


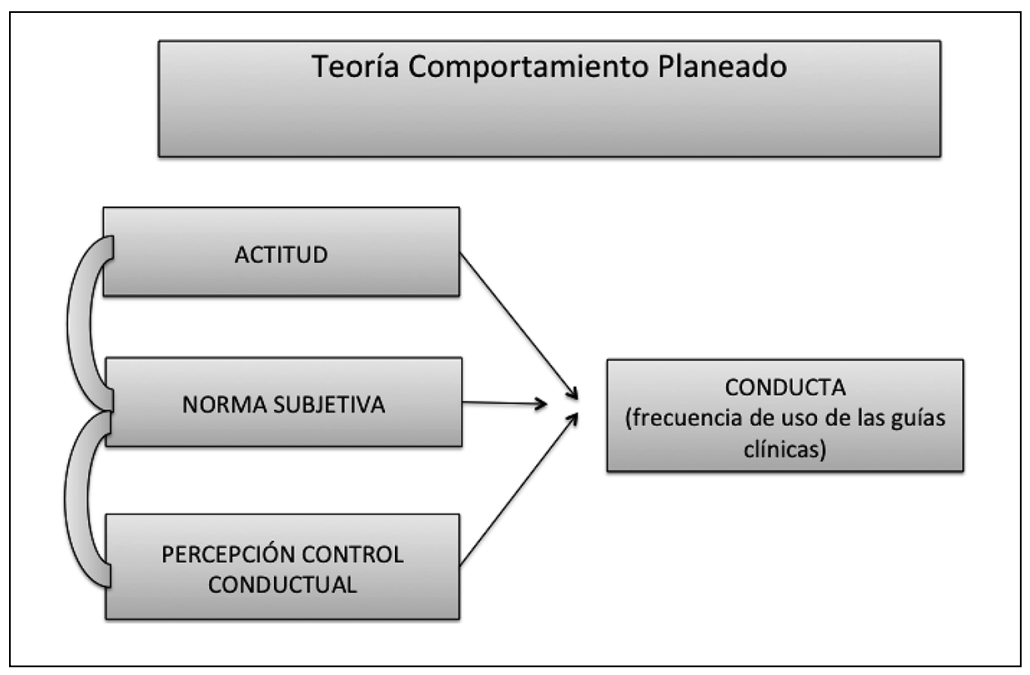

Figura 1. Teoría Comportamiento Planeado. obtuvieron datos de contacto de dichos médicos a través de los encargados de la implementación de GPC GES de Centros de Salud de AP a lo largo del país (información obtenida desde el Departamento Secretaria AUGE y de Coordinación Evidencial y Metodológica del MINSAL) y a través de contactos personales de los autores con diferentes Centros de Salud.

Se envió el cuestionario a los correos electrónicos utilizando el software Qualtrics, explicando el objetivo del estudio y garantizando el anonimato de las respuestas. Se enviaron recordatorios cada dos semanas por tres veces.

\section{Análisis}

Se realizó un análisis descriptivo de las variables, obteniéndose proporciones para variables categóricas y medias para variables continuas. Las diferencias entre las variables de la muestra y la población general de médicos se compararon utilizando la prueba estadística de chi-cuadrado para variables categóricas y la prueba t-test para variables continuas. Para explorar la asociación entre la "frecuencia de uso de las guías" (variable dependiente) con las variables demográficas y con las dimensiones de la TCP (actitud, norma subjetiva y control percibido) se realizaron dos análisis univariados utilizando pruebas de chi-cuadrado y t-test respectivamente. Finalmente, los potenciales predictores de la variable dependiente identificados a través del análisis univariado, fueron evalua- dos a través de un modelo de regresión logística. Se utilizó 5\% de nivel de significancia en todos los análisis, los que se realizaron con el programa estadístico SPSS v21.

El protocolo del estudio fue aprobado por el Comité de Ética de Investigación de la Escuela de Medicina de la Pontificia Universidad Católica de Chile.

\section{Resultados}

Se contactó por correo electrónico a 1.264 médicos, obteniéndose respuesta de 487 (38,5\%). De ellos, 354 (72,7\%) completaron el cuestionario en su totalidad, siendo los utilizados en el análisis. La tasa de respuesta efectiva fue de $28 \%$.

Al comparar las características de la muestra analizada con la base de datos de médicos de atención primaria del MINSAL, se encontraron diferencias significativas respecto de edad, género y nacionalidad (Tabla 1). La muestra analizada fue más joven, con mayor proporción de mujeres y de chilenos que la población de médicos de referencia. Sin embargo, no se encontraron diferencias significativas para el tipo de establecimiento (rural versus urbano) ni Región a la que pertenecían.

La mayoría de los que respondieron fueron médicos generales $(65 \%), 27 \%$ de médicos familiares y $8 \%$ de otra especialidad. $43,5 \%$ había trabajado en Atención Primaria entre 1-5 años, $26 \%$ realizaba labores docentes y $99 \%$ conocía 
Tabla 1. Características comparativas entre población de médicos y muestra obtenida

\begin{tabular}{|c|c|c|c|}
\hline Características & Muestra (n: 354) & Población (n: 3.831) & Valor $\mathbf{p}$ \\
\hline Edad (media) & 34,2 & 39,1 & $<0,05$ \\
\hline \multicolumn{4}{|l|}{ Género } \\
\hline Masculino & $44 \%$ & $49,6 \%$ & $<0,05$ \\
\hline Femenino & $56 \%$ & $50,3 \%$ & $<0,05$ \\
\hline \multicolumn{4}{|c|}{ Tipo establecimiento } \\
\hline Urbano & $80 \%$ & $77,2 \%$ & NS \\
\hline Rural & $20 \%$ & $22,8 \%$ & NS \\
\hline \multicolumn{4}{|l|}{ Región } \\
\hline Metropolitana & $42,6 \%$ & $43,3 \%$ & NS \\
\hline Otras Regiones & $57,4 \%$ & $56,7 \%$ & NS \\
\hline \multicolumn{4}{|l|}{ Nacionalidad } \\
\hline Chileno & $84,3 \%$ & $71,5 \%$ & $<0,05$ \\
\hline Extranjero & $15,7 \%$ & $28,5 \%$ & $<0,05$ \\
\hline
\end{tabular}

NS: no significativo.

las guías GES. De este último grupo, 97\% reportó que las utilizaba.

Respecto del dominio "frecuencia de uso de las guías", la mayoría de los médicos reportó que las usaban algunas veces, pero no todas las semanas (49\%) (Figura 2). Con respecto a qué parte de la guía es la más consultada, $82 \%$ respondieron que revisan los algoritmos, $50 \%$ las recomendaciones claves, $19 \%$ el texto extendido y $13 \%$ los anexos. Por otra parte, el formato más consultado fue la versión online ( $85 \%$ ) y la menos consultada fue la versión online impresa (4\%).
En cuanto a los aspectos que generan más confianza fueron que las guías incluyan clasificación de los niveles de evidencia $(70 \%)$ y fuerza de las recomendaciones (64\%). Por otra parte, la principal utilidad que se atribuye a las guías GES es que ayudarían a estandarizar y homogenizar la práctica asistencial (85\%). Respecto de la utilidad que les atribuyen a diferentes formas de difusión destacan la página web del MINSAL y la aplicación para teléfonos móviles. Las barreras más importantes para su uso fueron la falta de un formato breve, claro y de fácil acceso (63\%)

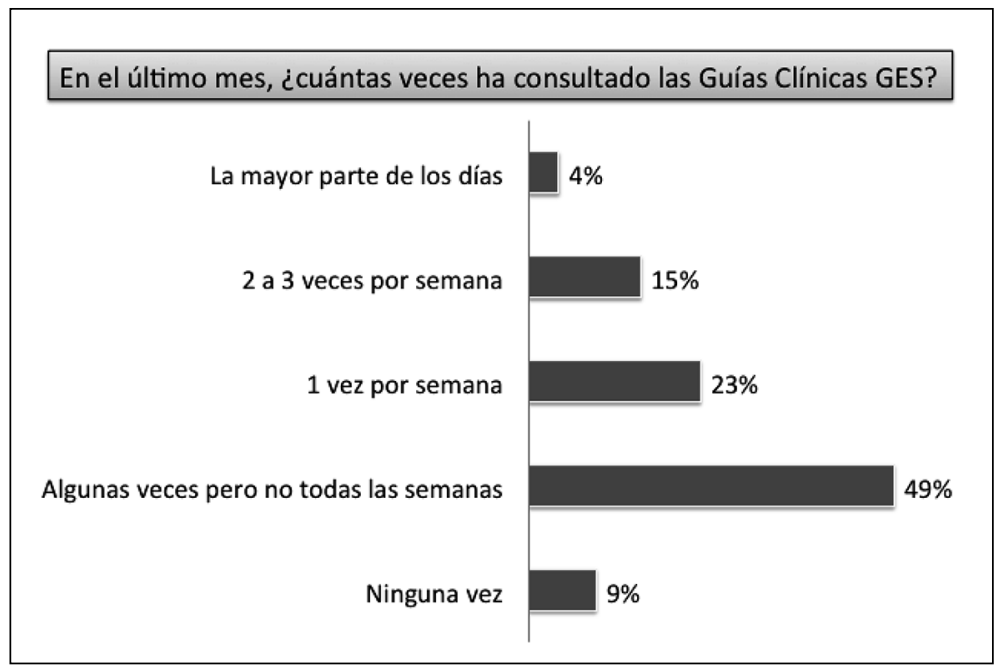

Figura 2. Frecuencia de uso de las GPC GES. 
y de tiempo para acceder a la información de la guía $(53 \%)$.

En el análisis univariado entre los datos demográficos y la "frecuencia de uso de las guías" se observó que médicos de menor edad $(<35$ años), que trabajan en la Región Metropolitana y de nacionalidad chilena parecen utilizar las guías con mayor frecuencia que los grupos de mayor edad, que trabajan en otras Regiones y de nacionalidad extranjera, respectivamente (Tabla 2). Al comparar los puntajes promedio de cada una de las dimensiones de la TCP en los grupos con diferentes frecuencias de uso de GPC se observó que para cada una de ellas (actitud, norma subjetiva y control percibido) los puntajes eran significati- vamente mayores en el grupo con alta frecuencia de uso comparado con el de baja frecuencia de uso (Tabla 3). Es decir, una actitud más positiva, una norma subjetiva más favorable y un mayor control percibido hacia las GPC se asociaron con una mayor frecuencia de uso de ellas.

El análisis multivariado mostró que -ajustando por el efecto de las otras variables- tanto actitud (OR 1,51, IC 1,07-2,13) como norma subjetiva (OR 1,46, IC 1,1-1,95) y control percibido (OR 1,42 IC 1,01-2) estuvieron asociadas con mayor frecuencia de uso, mientras que, la mayor edad (OR 0,93 , IC $0,89-0,97)$ y la nacionalidad extranjera (OR 0,42 , IC $0,21-0,84$ ) se asociaron a menor frecuencia de uso.

Tabla 2. Proporción de "Frecuencia de Uso de las Guías" con datos demográficos

\begin{tabular}{|c|c|c|c|}
\hline Características & $\begin{array}{l}\text { Alta frecuencia de uso } \\
\text { ( } \geq 1 \text { vez por semana) }\end{array}$ & $\begin{array}{l}\text { Baja frecuencia de uso } \\
\text { (< } 1 \text { vez por semana) }\end{array}$ & Valor $\mathbf{p}$ \\
\hline $\begin{array}{l}\text { Edad (media) } \\
\quad \geq 35 \text { años } \\
<35 \text { años }\end{array}$ & $\begin{array}{l}30,9 \% \\
46,6 \%\end{array}$ & $\begin{array}{l}69,1 \% \\
53,4 \%\end{array}$ & $<0,05$ \\
\hline $\begin{array}{l}\text { Género } \\
\text { Masculino } \\
\text { Femenino }\end{array}$ & $\begin{array}{l}47,3 \% \\
37,2 \%\end{array}$ & $\begin{array}{l}52,7 \% \\
62,8 \%\end{array}$ & NS \\
\hline $\begin{array}{l}\text { Tipo establecimient } \\
\text { Urbano } \\
\text { Rural }\end{array}$ & $\begin{array}{l}47,9 \% \\
40,1 \%\end{array}$ & $\begin{array}{l}52,1 \% \\
59,9 \%\end{array}$ & NS \\
\hline $\begin{array}{l}\text { Región } \\
\text { Metropolitana } \\
\text { Otras Regiones }\end{array}$ & $\begin{array}{l}33,3 \% \\
57,6 \%\end{array}$ & $\begin{array}{l}66,7 \% \\
42,4 \%\end{array}$ & $<0,05$ \\
\hline $\begin{array}{l}\text { Nacionalidad } \\
\text { Chileno } \\
\text { Extranjero }\end{array}$ & $\begin{array}{l}39 \% \\
13 \%\end{array}$ & $\begin{array}{l}61 \% \\
87 \%\end{array}$ & $<0,05$ \\
\hline
\end{tabular}

NS: no significativo.

Tabla 3. Asociación entre "Frecuencia de Uso de las Guías" con constructos de la TCP (actitud, norma subjetiva y control percibido)

\begin{tabular}{|lccc|}
\hline Características & $\begin{array}{c}\text { Alta frecuencia de uso } \\
\text { ( } \geq \mathbf{1} \text { vez por semana) }\end{array}$ & $\begin{array}{c}\text { Baja frecuencia de uso } \\
\text { (< 1 vez por semana) }\end{array}$ & Valor $\mathbf{p}$ \\
Actitud & $\overline{\mathbf{x}}$ & $\overline{\mathbf{x}}$ & $<0.05$ \\
Norma subjetiva & 4,97 & 4,5 & $<0.05$ \\
Control percibido & 4,52 & 4,02 & $<0.05$ \\
\hline
\end{tabular}




\section{Discusión}

Médicos de atención primaria conocen las GPC del Régimen GES, pero las utilizan en frecuencia variable, siendo las principales barreras para su uso la falta de tiempo y de un formato breve, claro y de fácil acceso. La sección más utilizada es la de algoritmos y la versión más utilizada es la online. Además, existe una percepción positiva de la diseminación de las GPC por medios electrónicos, como la página web del MINSAL y formatos compatibles con teléfonos móviles.

Dentro de los aspectos que dan credibilidad y confianza, los más frecuentemente reportados fueron aquellos relacionados con la inclusión de una clasificación de los niveles de evidencia y la fuerza de las recomendaciones y aquellos relacionados al equipo e institución que elabora la guía.

Las tres dimensiones de la TCP (actitud, norma subjetiva y control percibido), estuvieron asociadas a una mayor frecuencia de uso de guías.

En general, estos resultados están en línea con lo reportado por otros estudios acerca del tema. Las barreras identificadas concuerdan con las descritas en estudios internacionales en donde se plantea la importancia de un formato corto, sencillo y que incluya material escrito de información a pacientes. Además la falta de tiempo en la consulta parece ser un tema recurrente identificado como barrera para el uso de $\mathrm{GPC}^{9-12,16}$. La evaluación de la evidencia y la "reputación" de la institución elaboradora son aspectos que otros estudios también han identificado como relacionados a la credibilidad que los médicos asignan a las $\mathrm{GPC}^{16,21}$. La edad es un factor, que también ha sido previamente descrito, con una menor frecuencia de uso de GPC en aquellos médicos de mayor edad ${ }^{16,21,22}$. La asociación entre las dimensiones de la TCP y la intención del profesional de utilizar las GPC también ha sido descrita por estudios similares en la literatura internacional ${ }^{23,24}$.

Algunos de nuestros hallazgos ya han sido abordados por el programa de GPC del MINSAL. El uso de medios electrónicos para la diseminación de las GPC, no sólo a través de la página web del MINSAL, es algo que se ha comenzado a implementar con el diseño de aplicaciones para teléfonos móviles. Sin embargo, todavía es necesario avanzar en formatos "amigables" para presentar la información en dichas plataformas de manera que sean fácilmente utilizables por los clínicos ${ }^{15}$. Asímismo, se ha realizado un esfuerzo en mejorar aspectos metodológicos que le den credibilidad a las GPC, como es la adopción de la metodología GRADE (Grading of Recommendations Assessment, Development and Evaluation) para establecer los niveles de evidencia y fuerza de las recomendaciones ${ }^{25}$.

Considerando la menor frecuencia de uso de las GPC por algunos médicos de nacionalidad extranjera y considerando que $30 \%$ de los médicos de atención primaria son extranjeros, debieran considerarse estrategias específicas que permitan fortalecer su uso por este grupo.

Las tres dimensiones de la TCP estuvieron relacionada a frecuencia de uso de GPC. Para potenciar estas dimensiones se podrían plantear estrategias como: (i) ofrecer a los médicos información detallada, individualizada y personalizada especificando qué beneficios puede obtener del uso de guías; (ii) indagar acerca de estrategias utilizadas anteriormente para el uso de guías y que pueden permitir nuevas variaciones en la conducta; (iii) ofrecer retroalimentación acerca de otras situaciones que el médico haya podido resolver favorablemente con el uso de GPCs; (iv) líderes y autoridades locales que promuevan el uso de las GPC; (v) abordar las barreras percibidas y facilitar los recursos para el uso.

Dentro de las limitaciones de nuestro estudio, estuvo la dificultad para obtener un registro confiable de médicos de atención primaria. A pesar de ello, a través de diversas estrategias se logró contactar a un grupo importante de ellos. La tasa de respuesta no fue la óptima $(28 \%)$ a pesar de la aplicación de estrategias para aumentarla ${ }^{26,27}$. Sin embargo, esta tasa de respuesta se encuentra dentro del rango reportado por estudios similares en la literatura ${ }^{26,27}$. Ciertas características sociodemográficas de la muestra se pudieron comparar con la base de datos actualizada de médicos de atención primaria, resultando en diferencias que sugieren cautela en relación con la aplicabilidad y transferibilidad de nuestros hallazgos. Por ejemplo, el promedio de edad de la muestra fue menor que el de la población de médicos de atención primaria, por lo que el conocimiento de las guías y la frecuencia de uso podría ser aún menor de lo reportado.

Otra limitación estuvo relacionada a la validez del instrumento utilizado para la recolección de la 
información. A pesar de contar con un proceso de validación de sus propiedades de medición ${ }^{14,16}$, no realizamos un nuevo proceso de validación formal en nuestro contexto. Sin embargo, se realizaron pruebas piloto que permitieron, al menos, chequear su validez de apariencia.

En resumen, médicos de atención primaria conocen las GPC del Régimen de GES y las utilizan. Las dimensiones de la TCP pueden servir de base para el diseño de estrategias específicas que mejoren el uso de GPC. A pesar de que estudios similares se han realizado en otros países, este es el primer estudio realizado en nuestro país evaluando las percepciones y actitudes los médicos de atención primaria hacia GPC GES. Algunos hallazgos ya han sido incorporados en el programa de elaboración de GPC del MINSAL, pero todavía existe espacio para diseñar e implementar estrategias más específicas basadas en los resultados aquí presentados que mejoren el uso de las GPC GES y puedan contribuir a mejores resultados sanitarios en nuestros pacientes.

\section{Anexo 1. Cuestionario}

Q1 1. Año de nacimiento:

\section{Q2 2. Sexo}

O Masculino (1)

O Femenino (2)

Q35 3. Nacionalidad:

\section{Q3 4. ¿Cuál es su especialidad?}

O Medicina general (1)

Medicina familiar (2)

O Otra, por favor, especifique cuál: (3)

Q4 5. ¿Desde hace cuantos años trabaja en atención primaria?

O menos de 1 año (1)

O entre 1 - 5 años (2)

O entre 5 -10 años (3)

O más de 10 años (4)

Q5 6. ¿En qué ámbito y nivel asistencial realiza su actividad principal?

O Atención primaria - urbana (1)

O Atención primaria - rural (2)

Otros, por favor, especifique cuál: (3)

Q6 7. ¿En qué región del país trabaja?

Q7 8. ¿Realiza labores docentes en universidad?

$$
\text { OSi (1) }
$$$$
\text { ONo (2) }
$$

Q9 9. ¿Conoce la existencia de las Guías Clínicas GES? OSi (1)

O No [En este caso no es necesario continuar con la encuesta] (2)
Q8 10. ¿Ha participado como miembro de algún grupo de elaboración de una Guía Clínica GES?

OSi (1)

ONo (2)

Q12 11. ¿Ha actuado como revisor externo de una Guía Clínica GES?

OSi (1)

O No (2)

Q13 12. ¿Utiliza las Guías Clínicas GES?

OSi (1)

O No [En este caso no es necesario continuar con la encuesta] (2)

Q10 13. En el último mes, ¿cuántas veces ha consultado las Guías Clínicas GES?

O La mayor parte de los días de la semana (1)

ODos o tres veces por semana (2)

OUna vez a la semana (3)

OAlguna vez, pero no todas las semanas (4)

O Ninguna vez (5)

Q11 14. Ante una duda clínica, cuando consulta una Guía Clínica GES, ¿qué parte de la guía clínica es la(s) que más revisa(s) habitualmente? (Puede marcar más de una respuesta)

OAlgoritmos (1)

O Recomendación/es claves (2)

OTexto extendido (3)

OAnexos (4)

Q14 15. A la hora de consultar una Guía Clínica GES, habitualmente utiliza:

O La versión online (1)

O La versión online impresa (2)

O La versión original impresa de la guía (3)

OTodas por igual (4) 
Q15 16. ¿Qué utilidad le atribuiría a cada una de las siguientes formas de difusión de las Guías Clínicas GES? Marque una opción en una escala de 1 al 7, siendo 1 nada de útil y 7 muy útil.

\begin{tabular}{|c|c|c|c|c|c|c|c|}
\hline & $\begin{array}{l}1 \text { Nada } \\
\text { útil (1) }\end{array}$ & $2(2)$ & $3(3)$ & $4(4)$ & $5(5)$ & $6(6)$ & $\begin{array}{l}7 \text { Muy } \\
\text { útil (7) }\end{array}$ \\
\hline $\begin{array}{l}\text { Folleto informativo del Ministerio } \\
\text { de Salud (1) }\end{array}$ & O & O & $\mathrm{O}$ & $\mathrm{O}$ & O & O & O \\
\hline Sociedades Científicas (2) & $\mathrm{O}$ & $\mathrm{O}$ & $\mathrm{O}$ & O & O & O & $\mathrm{O}$ \\
\hline Congreso/Jornada Científica (3) & $\mathrm{O}$ & $\mathrm{O}$ & $\mathrm{O}$ & $\mathrm{O}$ & $\mathrm{O}$ & $\mathrm{O}$ & $\mathrm{O}$ \\
\hline $\begin{array}{l}\text { Reunión clínica en el servicio o } \\
\text { el centro de salud (4) }\end{array}$ & $\mathrm{O}$ & $\mathrm{O}$ & O & $\mathrm{O}$ & O & O & $\mathrm{O}$ \\
\hline $\begin{array}{l}\text { Artículo resumiendo una o más } \\
\text { guías (5) }\end{array}$ & O & $\mathrm{O}$ & O & $\mathrm{O}$ & $\mathrm{O}$ & O & $\mathrm{O}$ \\
\hline Aplicación en smartphone (6) & $\mathrm{O}$ & $\mathrm{O}$ & O & 0 & O & $\mathrm{O}$ & $\mathrm{O}$ \\
\hline $\begin{array}{l}\text { Tríptico de bolsillo con un } \\
\text { resumen de la guía ( } 7 \text { ) }\end{array}$ & O & $\mathrm{O}$ & O & $\mathrm{O}$ & O & O & O \\
\hline Página web del MINSAL (8) & $\mathrm{O}$ & $\mathrm{O}$ & O & O & $\mathrm{O}$ & O & O \\
\hline Guía impresa (10) & $\mathrm{O}$ & $\mathrm{O}$ & $\mathrm{O}$ & $\mathrm{O}$ & O & $\mathrm{O}$ & $\mathrm{O}$ \\
\hline Otra (especifíque cual) (9) & $\mathrm{O}$ & $\mathrm{O}$ & $\mathrm{O}$ & $\mathrm{O}$ & $\mathrm{O}$ & $\mathrm{O}$ & $\mathrm{O}$ \\
\hline
\end{tabular}

Q17 17. Valore cada uno de los siguientes enunciados marcando la opción que más se adecue a su sentir u opinión. Marque una opción en una escala de 1 al 7, siendo 1 totalmente en desacuerdo y 7 totalmente de acuerdo. ACTITUD

\begin{tabular}{|c|c|c|c|c|c|c|c|}
\hline & $\begin{array}{l}1 \text { Totalmente } \\
\text { en desacuerdo } \\
(1)\end{array}$ & $2(2)$ & $3(3)$ & $4(4)$ & $5(5)$ & $6(6)$ & $\begin{array}{l}7 \text { Totalmente } \\
\text { de acuerdo } \\
\text { (7) }\end{array}$ \\
\hline $\begin{array}{l}\text { Cuando uso las guías creo que } \\
\text { estoy haciendo algo positivo por } \\
\text { el/la paciente (1) }\end{array}$ & 0 & 0 & 0 & 0 & 0 & 0 & 0 \\
\hline $\begin{array}{l}\text { El seguimiento de las guías me } \\
\text { ayuda a diagnosticar mejor ( } 2 \text { ) }\end{array}$ & 0 & O & O & O & O & O & 0 \\
\hline $\begin{array}{l}\text { Si uso las guías tengo que ver más } \\
\text { veces a el/la paciente (3) }\end{array}$ & 0 & 0 & 0 & 0 & 0 & 0 & 0 \\
\hline $\begin{array}{l}\text { Si sigo las guías se mejora en ge- } \\
\text { neral la salud de la población (4) }\end{array}$ & 0 & 0 & 0 & 0 & 0 & 0 & 0 \\
\hline $\begin{array}{l}\text { Si sigo las guías aconsejo mejor a } \\
\text { el/la paciente (5) }\end{array}$ & 0 & O & O & 0 & O & 0 & 0 \\
\hline $\begin{array}{l}\text { Con el uso de las guías los/las } \\
\text { pacientes se involucran más en } \\
\text { la toma de decisiones sobre su } \\
\text { salud (6) }\end{array}$ & 0 & 0 & 0 & 0 & 0 & 0 & 0 \\
\hline $\begin{array}{l}\text { Con las guías se hace mejor el se- } \\
\text { guimiento de los/las pacientes (7) }\end{array}$ & 0 & 0 & 0 & 0 & 0 & 0 & 0 \\
\hline $\begin{array}{l}\text { Las guías reducen la autonomía } \\
\text { del médico (8) }\end{array}$ & 0 & 0 & 0 & 0 & 0 & 0 & 0 \\
\hline $\begin{array}{l}\text { Los/las pacientes se sienten } \\
\text { incómodos/as cuando consulto las } \\
\text { guías delante de ellos/ellas (9) }\end{array}$ & 0 & 0 & O & O & 0 & 0 & 0 \\
\hline $\begin{array}{l}\text { Aplicar las guías me proporciona } \\
\text { seguridad en la atención (10) }\end{array}$ & 0 & 0 & 0 & 0 & 0 & 0 & 0 \\
\hline
\end{tabular}




\begin{tabular}{|c|c|c|c|c|c|c|c|}
\hline $\begin{array}{l}\text { La eficacia de la consulta disminu- } \\
\text { ye con la aplicación de las guías } \\
\text { (11) }\end{array}$ & 0 & O & 0 & 0 & $\mathrm{O}$ & O & 0 \\
\hline $\begin{array}{l}\text { Seguir las guías facilita la coordi- } \\
\text { nación entre las distintas especiali- } \\
\text { dades (12) }\end{array}$ & $\mathrm{O}$ & $\mathrm{O}$ & O & O & $\mathrm{O}$ & $\mathrm{O}$ & O \\
\hline
\end{tabular}

Q20 18. ¿Qué grado de confianza le generan las Guías Clínicas GES?

\begin{tabular}{|l|c|c|c|c|c|c|c|}
\hline & $\begin{array}{c}1 \text { Ninguna } \\
\text { confianza } \\
(1)\end{array}$ & $2(2)$ & $3(3)$ & $4(4)$ & $5(5)$ & $6(6)$ & $\begin{array}{c}7 \text { Total con- } \\
\text { fianza (7) }\end{array}$ \\
\hline.$(1)$ & 0 & 0 & 0 & 0 & 0 & 0 & 0 \\
\hline
\end{tabular}

Q21 19. ¿Cuáles son los aspectos principales de ésta que le generan más confianza? [Puede marcar más de una respuesta] O La metodología de elaboración (1)

O La redacción (2)

O Que incluya la clasificación de los niveles de evidencia (3)

O Que incluya una clasificación de los grados o fuerza de las recomendaciones (4)

O La institución o promotor de la guía (5)

$\mathrm{O}$ La independencia en la fuente de financiamiento (6)

O El equipo elaborador (7)

La declaración de conflicto de interés de los autores (8)

O Ninguno (9)

Otro, por favor, especifique cuál (10)

\section{Referencias}

1. Institute of Medicine (US), Committee on Standards for Developing Trustworthy Clinical Practice Guideline. Clinical Practice Guidelines We Can Trust. Washington, DC: The National Academies Press, 2011.

2. García JF, Bravo R. Guías de práctica clínica en Internet. Aten Primaria 2001; 28(1): 74-9.

3. Clancy C, Cronin K. Evidence-Based Decision Making: Global evidence, Local decisions. Health Affairs 2005; 24(1): 151-62.

4. Scottish Intercollegiate Guidelines Network. Sign 50: A guideline developers' handbook. [Internet] Edinburg: SIGN; 2008.

5. Unidad de Evaluación de Tecnologías de Salud. Departamento de Calidad de Prestadores. División de Planificación y Presupuesto, Ministerio de Salud. Pauta para la Elaboración, Aplicación y Evaluación de Guías de Práctica Clínica. 2002. https://www.minsal.cl/portal/ url/item/6d054172ffd27defe04001011f010ee8.pdf.

6. Atienza G, Bañeres J, Gracia FJ. Guías de práctica clínica y atención primaria. Informe SESPAS 2012. Gac Sanit 2012; 26 (1): 113-7.
7. Fonarow GC, Yancy CW, Hernández AF, Peterson ED, Spertus JA, Heidenreich PA. Potential impact of optimal implementation of evidence-based heart failure therapies on mortality. Am Heart J 2011; 161 (6): 1024-30.

8. Lemp S, Calvo E. ¿Aceptación o rechazo de la estandarización clínica?: Médicos chilenos hablan de las guías clínicas y canastas de prestaciones. Salud colectiva [online] 2012; 8(1): 61-8.

9. Saillour-Glenisson F, Michel P. Individual and collective facilitators of and barriers to the use of clinical practice guidelines by physicians: a literature review. Rev Epidemiol Sante Publique 2003; 51 (1): 65-80.

10. Farquhar C, Kofa E, Slutsky J. Clinicians' attitudes to clinical practice guidelines: a systematic review. Med J Aust 2002; 177 (9): 502-6.

11. Carlsen B, Glenton C, Pope C. Thou shalt versus thou shalt not: a meta-synthesis of GPs' attitudes to clinical practice guidelines. Br J Gen Pract 2007; 57 (545): 971-8.

12. Lugtenberg $M$, Zegers-Van Schaick J, Westert G, Burgers J. Why don't physicians adhere to guideline recommendations in practice? An analysis of barriers among Dutch general practitioners. Implement Sci 2009; 4:54. 
13. Formoso G, Liberati A, Magrini N. Practice guidelines: useful and "participative" method? Survey of Italian physicians by professional setting. Arch Intern Med 2001; 161 (16): 2037-42.

14. Kotzeva A, Solà I, Carrasco J, Díaz del Campo P, Gracia F, Calderón E, et al. Perceptions and attitudes of clinicians in Spain toward clinical practice guidelines and grading systems: a protocol for a qualitative study and a national survey. BMC Health Serv Res 2010; 10: 328.

15. Ministerio de Salud. Manual Metodológico. Desarrollo de Guías de Práctica Clínica. Santiago: Gobierno de Chile, 2014. http://www.bibliotecaminsal.cl/wp/wp-content/ uploads/2016/04/Manual-metodologico-GPC-151014. pdf.

16. Kotzeva A, Guillamón I, Gracia J, Díaz del Campo P, Gich I, Calderón E, et al. Use of clinical practice guidelines and factors related to their uptake: a survey of health professionals in Spain. J Eval Clin Pract 2014; 20 (3): 216-24.

17. Ajzen I, The theory of planned behavior. Organizational Behavior and Human Decision Processes 1991; 50: 179211.

18. Glanz K, Rimer B, Viswanath K, editors. Health Behavior and Health Education: Theory, Research, and Practice (4th ed). San Francisco, USA: Jossey-Bass; 2008.

19. Armitage C, Conner M. Efficacy of the Theory of Planned Behaviour: A Meta-Analytic Review. Br J Soc Psychol 2001; 40 (4): 471-99.

20. Albarracín D, Johnson B, Fishbein M, Muellerleile P. Theories of Reasoned Action and Planned Behavior as
Models of Condom Use: A Meta-analysis. Psychol Bull 2001; 127(1): 142-61.

21. Shea A, DePuy V, Allen J, Weinfurt K. Use and perceptions of clinical practice guidelines by internal medicine physicians. Am J Med Qual 2007; 22 (3): 170-6.

22. Flores G, Lee M, Bauchner H, Kastner B. Pediatricians' attitudes, beliefs, and practices regarding clinical practice guidelines: a national survey. Pediatrics 2000; 105 (3): 496-501.

23. Kortteisto T, Rissanen P. Healthcare professionals' intentions to use clinical guidelines: a survey using the theory of planned behaviour. Implement Sci 2010; 5:51.

24. Côté F, Gagnon J, Houme P, Abdeljelil A, Gagnon M. Using the Theory of Planned Behaviour to predict nurses' intention to integrate research evidence into clinical decision-making. J Adv Nurs 2012; 68 (10): 2289-98.

25. Alonso-Coello P, Schünemann J, Moberg J, Brignardello-Petersen R, AklElie A, Davoli M, et al. GRADE Evidence to Decision (EtD) frameworks: a systematic and transparent approach to making well informed healthcare choices. 1: Introduction. BMJ 2016; 353: i2016.

26. Cho Y, Johnson T, VanGeest J. Enhancing Surveys of Health Care Professionals: A Meta-Analysis of Techniques to Improve Response. Eval Health Prof 2013; 36 (3): 382-407.

27. VanGeest J, Johnson T, Welch V. Methodologies for Improving Response Rates in Surveys of Physicians: A Systematic Review. Eval Health prof 2007; 30 (4): 30321. 